\title{
Manejo de la mosca blanca con productos botánicos y químicos en tomate, en Estelí, Nicaragua
}

\author{
Management of whitefly with botanical and chemical products in tomato, in Esteli, Nicaragua
}

Edgardo Jiménez Martínez ${ }^{1}$

Harlin Demetrio García Cruz ${ }^{2}$

\section{Resumen}

La mosca blanca (Bemisia tabaco, especie de hemíptero esternorrinco de la familia Aleyrodidae) y los geminivirus son los principales agentes de daño fitosanitarios severos para los productores de tomate (Solanum lycopersicum L.), en el municipio de Estelí. Esta plaga provoca grandes pérdidas económicas, ha disminuido los rendimientos y afecta la calidad de los frutos, lo cual incurre en mayores costos de producción. Con base en esta problemática, en el municipio de Estelí se realizó un estudio cuantitativo del tipo experimental, del o8 de octubre al 11 de diciembre 2018, con el objetivo de evaluar alternativas químicas y botánicas para el manejo del complejo mosca blancaGeminivirus, para esto se estableció un experimento usando un diseño de bloques completos al azar (BCA), con seis tratamientos; T1. Evisect ${ }^{\circledR}, \mathrm{T}_{2}$. Epingle ${ }^{\circledR}, \mathrm{T}_{3}$. Kabonim ${ }^{\circledR}$, T4. Savon ${ }^{\circledR}, \mathrm{T}_{5}$. Quamar/Bioquamar ${ }^{\circledR}$ y T6. Testigo (agua), en este estudio se empleó la variedad Orna, las variables que se evaluaron fueron: número de Bemisia tabaci por planta, incidencia y severidad de virosis, rendimiento de tomate en $\mathrm{kg} / \mathrm{ha}^{1}$ de los tratamientos evaluados, análisis económico (parcial).

De acuerdo a los resultados, los tratamientos que presentaron menor porcentaje de daño de Bemisia tabaci por planta fueron: Savon ${ }^{\circledR}$, Epingle ${ }^{\circledast}$, con respecto a incidencia y severidad de virosis, los tratamientos con mejores resultados fueron: Savon $^{\circledR}$, Quamar ${ }^{\circledast}$ y Kabonim ${ }^{\circledast}$. El análisis económico (parcial) realizado determinó que los mejores rendimientos fueron Epingle ${ }^{\circledR}$ con $44,057.77 \mathrm{~kg} / \mathrm{ha}^{1}$ y Kabonim ${ }^{\circledR}$ con $34,469.70 \mathrm{~kg} / \mathrm{ha}^{1}$.

Palabras claves: incidencia, severidad, Orna, Bemisia tabaci, Geminivirus.

\section{Abstract}

The whitefly (Bemisia tabaco, a species of sternorrhine hemiptera of the family Aleyrodidae) and geminiviruses are the main agents of severe phytosanitary damage to tomato (Solanum lycopersicum L.) producers in the municipality of Estelí. This pest causes

\footnotetext{
1 Ing. MSc. Sanidad Vegetal, Universidad Nacional Agraria, Nicaragua, Correo: hadegarcruz@gmail.com, ORCID: https://orcid. org/oooo-0002-7224-2223

2 Ing. MSc. Sanidad Vegetal, Universidad Nacional Agraria, Nicaragua, Correo: hadegarcruz@gmail.com, ORCID: https://orcid. org/0000-0002-7224-2223
}

Recibido: 24/08/2021 - Aprobado: 22/11/2021 


\section{AGROPECUARIA}

great economic losses, has reduced yields and affects fruit quality, which incurs higher production costs. Based on this problem in the municipality of Estelí, a quantitative study of the experimental type was conducted from October 8th to December 11th, 2018, with the objective of evaluating chemical and botanical alternatives for the management of the whitefly-Geminivirus complex, for this an experiment was established using a randomized complete block design (BCA), with six treatments; T1. Evisect ${ }^{\circledR}$, T2. Epingle ${ }^{\circledR}, \mathrm{T}_{3}$. Kabonim ${ }^{\circledR}, \mathrm{T}_{4}$. Savon ${ }^{\circledR}$, T5. Quamar/Bioquamar ${ }^{\circledR}$ and T6. Control (water), the Orna variety was used in this study. The variables evaluated were: number of Bemisia tabaci per plant, incidence and severity of virosis, tomato yield in $\mathrm{kg} / \mathrm{ha}^{1}$ of the treatments evaluated, economic analysis (partial). According to the results, the treatments with the lowest percentage of Bemisia tabaci damage per plant were: Savon $^{\circledast}$, Epingle ${ }^{\circledast}$, with respect to incidence and severity of virosis, the treatments with the best results were: Savon ${ }^{\circledR}$, Quamar ${ }^{\circledR}$ and Kabonim ${ }^{\circledR}$. The economic analysis (partial) carried out determined that the best yields were Epingle ${ }^{\circledR}$ with $44,057.77 \mathrm{~kg} /$ $\mathrm{ha}^{1}$ and Kabonim ${ }^{\circledR}$ with $34,469.70 \mathrm{~kg} / \mathrm{ha}^{1}$.

Keywords: incidence, severity, Orna, Bemisia tabaci, Geminivirus.

\section{Introducción}

El cultivo del tomate, desde la antigüedad ha sido la dieta del ser humano, así también una de las hortalizas más cultivada en todo el mundo, es originario de las regiones andinas y se extiende desde el sur de Colombia hasta el norte de Chile, desde donde posiblemente fue llevado a Centroamérica y México, donde se comenzó a cultivar.

Nicaragua posee gran potencial para la producción de hortalizas frescas para el mercado interno y de exportación, contando con una privilegiada ubicación geográfica con respecto a otros países y variedad de ambientes; la horticultura está concentrada en la región norcentral, implementada por pequeños y medianos productores. (Instituto Interamericano de Cooperación para la Agricultura [IICA], 2004, p. 7)

La producción de tomate se encuentra acorde con la demanda nacional, el variado comercio ha proporcionado diversas variedades, los productores se encuentran interesados en aplicar nuevas y mejores técnicas de producción que les garanticen mayores rendimientos, pero también mejor calidad. (El Nuevo Diario, 2007, p. 2).

"Aunque hay gran cantidad de variedades que se ha generado y comercializado de este cultivo, la mayoría son susceptibles a una variedad amplia de plagas de artrópodos, capaces de ocasionar daños, incluida la destrucción completa del cultivo" (Foolad, 2012, p. 5). 
Existen diversas variedades y tipos de tomates, uno de los más cultivados en Nicaragua es el criollo o de uso industrial, dentro del cual está el cultivar Orna F1, Orna es una planta de porte determinado y producción precoz, con resistencia a Virus TSWV, TYLCV y tolerancia Pseudomonas sp, Xhantomonas sp, Alternaria sp y Phytophtora sp, Nematodos (Meloidogyne sp, Pratylenchus sp). (Hazera Genetics, 2015a, p. 1)

\section{Materiales y métodos}

\section{Ubicación del área de estudio}

El estudio se realizó en la finca "Villa Vieja", propiedad del Instituto Técnico Agropecuario de la Diócesis de Estelí (ITADE) en el municipio de Estelí, departamento de Estelí, ubicada en las coordenadas geográficas latitud norte de $13^{\circ} 05^{\prime} 30.7^{\prime \prime}$ y $86^{\circ} 21^{\prime} 13.8^{\prime \prime}$ de longitud oeste, a una altitud de $837 \mathrm{msnm}$, con una extensión territorial de 796 $\mathrm{km}^{2}$, y una población de 128,761 habitantes, temperaturas medias anuales de $22.3^{\circ} \mathrm{C}$ y con precipitación entre $924 \mathrm{~mm}$. (Instituto Nicaragüense de Fomento Municipal [INIFOM], 2000, p.1)

\section{Establecimiento del ensayo}

El ensayo se llevó a cabo del 4 de octubre al 11 de diciembre del 2018. La investigación es cuantitativa del tipo experimental (Bloques Completos al Azar, BCA). Se estableció semillero bajo condiciones de invernadero, usando bandejas plásticas de 128 alveolos donde se depositaron las semillas. Una vez que las plantas alcanzaron los 18 días después de la germinación, fueron trasplantadas. La variedad del tomate utilizada fue híbrida "Orna", con resistencia a virus TSWV, TYLCV, el cual tiene tolerancia: Pseudomonas sp, Xhantomonas sp. Alternaria sp y Phytophtora sp y a nematodos como Meloidogyne sp, Pratylenchus sp.

Este híbrido tolera climas cálidos, tiene fruto tipo saladette (Roma), con peso que va de $150 \mathrm{~g}$ a $18 \mathrm{og}$ por fruto, con resistencia a transporte, excelente durabilidad, con coloración rojo intenso, con rendimiento promedio de $57,000 \mathrm{~kg} / \mathrm{ha}^{-1}$, con excelente respuesta a aplicaciones y fertilizantes. (Hazera Genetics, 2015b, p.1)

El semillero se estableció bajo condiciones protegidas (túnel), donde se realizó el manejo contra hongos fitopatógenos con carbendazim ${ }^{\circledR}$ y phyton ${ }^{\circledR}$. La preparación del terreno fue de forma mecánica y el trasplante se realizó a los 18 días después de sembradas las semillas en bandejas de polietileno.

El tutoreo se realizó a los 28 días después del trasplante, utilizando estacas a una distancia de $1.5 \mathrm{~m}$ entre cada una y nylon para sostener la planta, esta actividad se ejecutó en tres ocasiones durante cada ciclo del cultivo, la fertilización fue desarrollada 
por cada etapa fenológica de la planta, durante los primeros 10 días después del trasplante 18-46-0, segunda a los 25 días, 12-24-12, tercera y última a los 40 días con la fórmula o-o-6o.

\section{Diseño experimental}

El área total del experimento fue de $745 \mathrm{~m}^{2}$. El estudio se estableció bajo un diseño de $\mathrm{BCA}$, con cuatro repeticiones por tratamiento, donde se evaluaron dos tratamientos químicos y tres botánicos, más un testigo absoluto (agua), para el manejo de los insectos plagas del tomate.

\section{Descripción de los tratamientos evaluados}

\section{Tratamiento 1. Evisect ${ }^{\circledast}$}

Es un insecticida sistémico de origen biológico del grupo de las nereistoxinas, (Thiocyclam hidrogenoxalato), de acción sistémica en la planta, actúa por contacto e ingestión y se distribuye de forma acrópeta, incrementándose su actividad en control de los insectos. Al ser ingerido, este producto se transforma en el intestino de los insectos en nereistoxinas, sustancia que actúa en forma muy parecida a la nicotina, lo cual impide la transmisión de impulsos entre las células nerviosas. (Arysta, 2014, p. 1)

\section{Tratamiento 2. Epingle ${ }^{\oplus}$}

Su nombre común es Pyriproxyfen, con una concentración de1oo g i.a./L, su formulación es Concentración Emulsionable. Excelente herramienta para el MIP es sistémico local o de efecto translimitar (clave en el control de Ninfas), está clasificado por EPA como un insecticida de bajo impacto al medio ambiente, que inhibe la eclosión de los huevecillos (embriogénesis), interrumpe el desarrollo de los estadios ninfales (inhibición de metamorfosis) y suprime el desarrollo de huevecillos que ovipositan adultos tratados (Efecto Transovarial). (Sumitomo, 2008, p. 4)

\section{Tratamiento 3. Kabonim ${ }^{\circledast}$}

La composición del Kabonim ${ }^{\circledR}$ es un extracto de neem potásico (Azadirachtina indica) en un $40 \%$ y acondicionadores en $60 \%$ respectivamente. Es un insecticida de amplio espectro que controla insectos de cuerpo blando y duro interfiriendo con la hormona mudadora ecdisona. Sus ingredientes activos controlan las larvas, ninfas y estados de (puparios) mediante la interferencia en los metabolismos de las plagas. Controla y repele la mayor cantidad de insectos. (Atlántica, 2010a, p. 1) 


\section{Tratamiento 4. Savon ${ }^{\circledR}$}

Sus ingredientes activos son sales potásicas de ácidos grasos de aceite de almendras 50\% y acondicionadores 50\%, su nombre químico es Alquil Bencil Sulfonato, Metasilicato de Sodio, ácidos grasos, es de origen vegetal. Es un insecticida, acaricida y actúa por contacto, principalmente en insectos de cuerpo blando y larvas recién eclosionadas. Debe aplicarse, desde el momento en que aparecen los insectos en el cultivo, cubriendo completamente el haz y el envés de las hojas, según su formulación tiene toxicidad EPA: III. (Atlántica, 2010b, p. 3)

\section{Tratamiento 5. Quamar/Bioquamar ${ }^{\circledast}$}

Está compuesto por extracto de Quassia amara al 75\%. Su forma de acción es por contacto e ingestión, lo que provoca un trastorno en el sistema nervioso y sensorial, lo que hace disminuir la capacidad para la búsqueda de alimento. Por poseer un sabor extremadamente amargo le permite tener un efecto repelente y tóxico hacia los insectos. Provoca deshidratación a los insectos de cuerpo blando, ya sean adultos o inmaduros. Tiene la ventaja de ser un producto de origen orgánico, biodegradable y no contaminante. La dosis que se utiliza es de 0.5 a 0.75 litros por cada 200 litros de agua. (Atlántica, 2010c, p. 4)

\section{Tratamiento 6. Testigo absoluto / Consistió solamente en aplicación de agua.}

\section{Variables evaluadas}

\section{Número de Bemisia tabaci por planta (insectos/planta)}

"Se seleccionaron 25 plantas de la parcela al azar, utilizando hojas de muestreo para la recolección de datos. Los datos fueron recolectados semanalmente y los muestreos se realizaron por la mañana" (Programa de Manejo Integrado de Plagas para América Central [PROMIPAC], 2006, p. 37).

\section{Incidencia del daño por virosis transmitido por Bemisia tabaci}

Es la frecuencia con que las plantas presentaron síntomas del daño de virosis, o sea, el porcentaje de plantas con síntomas de daños con relación al número de plantas muestreadas; para diferenciar una planta sana de una enferma se hizo a través de la observación del síntoma característico de virosis trasmitido por Bemisia tabaci.

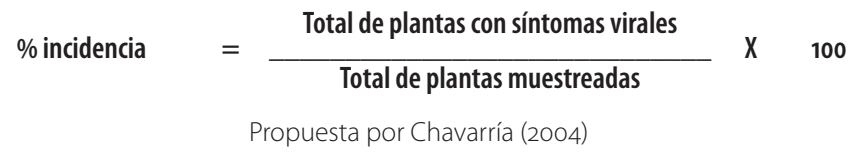




\section{Severidad de daño de virosis trasmitido por Bemisia tabaci}

Fue medida por el porcentaje de tejido afectado en una planta de tomate a través de la siguiente fórmula:

$$
\% \text { incidencia }=\quad \frac{\sum \text { de los grados de severidad encontrados }}{\text { Número de plantas muestreadas } \mathrm{x} \mathrm{grado} \mathrm{más} \mathrm{alto} \mathrm{de} \mathrm{la} \mathrm{escala}} \quad{ }^{100}
$$

Cuadro 1: Escala de severidad

\begin{tabular}{|ll|}
\hline \multicolumn{1}{|c|}{ Grados } & \multicolumn{1}{c|}{ Severidad síntomas } \\
\hline $0=0$ & No hay síntomas \\
$1=25$ & Débil mosaico y rizado en la lámina foliar de las hojas nuevas \\
$2=50$ & Mosaico y rizado de las hojas generalizado. \\
$3=75$ & Mosaico, rizado y deformación de hojas y ramas. \\
$4=100$ & Enanismo y deformación severa \\
\hline
\end{tabular}

Con el fin de facilitar la realización de este estudio, se utilizó una escala para medir el nivel de daño foliar causado por virosis en tomate (Jiménez Martínez, 2012).

\section{Rendimiento de tomate en $\mathrm{kg} / \mathrm{ha}^{1}$ de los tratamientos evaluados}

Este cálculo se hizo una vez terminada la cosecha. Se realizaron dos pases por semana, recogiendo solamente los frutos sin daño ocasionado por plagas y luego se pesaron para obtener el dato de peso total por cada uno de los tratamientos del estudio. Con los datos obtenidos se hizo una relación de rendimiento del tomate en una hectárea y se evaluó cuál de los tratamientos es el más rentable, tomando en cuenta los costos de producción y la ganancia obtenida a través de la comercialización.

\section{Análisis económico}

Se determinó cuál de los tratamientos tiene mejor retorno económico, de esta manera se identificó cuál es el de mejor rentabilidad tomando en cuenta la relación beneficio-costo, este análisis fue siguiendo la metodología donde se consideran diferentes costos, rendimientos y beneficio. (Centro Internacional de Mejoramiento de Maíz y Trigo [CIMMYT], 1988, p. 47)

Se tomaron los datos de rendimiento promedio $(R x)$ por tratamiento para la obtención del rendimiento ajustado (Rajust $=10 \% d e R x$ ), luego se calculó el beneficio bruto multiplicando el Rajust por el precio de venta en campo. La sumatoria de los costos totales, se estimó los costos de los insecticidas evaluados más el costo de aplicación del insecticida. Para costos fijos se incluyó la depreciación de equipos usados, mano 
de obra, control de plagas y enfermedades, etc. La obtención del beneficio neto, se restó los costos variables menos los costos fijos de cada tratamiento.

\section{Análisis de datos}

Una vez recolectados los datos, se ordenaron por variable y tratamiento, luego se realizó análisis con el modelo ANDEVA, de medidas repetidas en el tiempo. A los promedios de los tratamientos se les efectuó una comparación por medio de la prueba de separación de medias utilizando Tukey a un nivel de significancia de $P \leq 0.05$. A los promedios se les realizó una transformación de datos para las variables Incidencia y Severidad (\%) mediante la fórmula $\boldsymbol{\theta}=$ arcsen $\sqrt{ }(\mathbf{P})$. Para lo cual se utilizó el programa InfoStat. "Se realizó comparación de los rendimientos, a los que se les determinó la rentabilidad de los tratamientos, sometiendo los datos a análisis de presupuesto parcial" (Di Rienzo et al., 2017).

\section{Resultados y discusión}

\section{Fluctuación poblacional de Bemisia tabaci, en el cultivo de tomate en tratamientos evaluados de octubre a diciembre del 2018, Estelí}

Se comparó las fluctuaciones de Bemisia tabaci en parcelas tratadas con insecticidas botánicos, químicos y un testigo, a partir del 4 de octubre hasta el 11 de diciembre 2018. En este periodo se observó que la Bemisia tabaci se presentó en todos los tratamientos.

Se presentan dos poblacionales: uno a los $35 \mathrm{ddt}$ (5 de noviembre), presentando las poblaciones más bajas Savon ${ }^{\circledR}$, Quamar $^{\circledR}$ y Kabonim ${ }^{\circledR}$ con 33.5, 35.5 y 44.75 Bemisia tabaci por planta; el segundo poblacional se presentó a los 57 ddt (27 de noviembre), presentando las poblaciones menores, los insecticidas botánicos: Quamar ${ }^{\circledR}$, Savon $^{\circledR}$, y Kabonim $^{\circledR}$ con 251.25, 258 y 302.25 respectivamente Bemisia tabaci por planta (Figura 1).

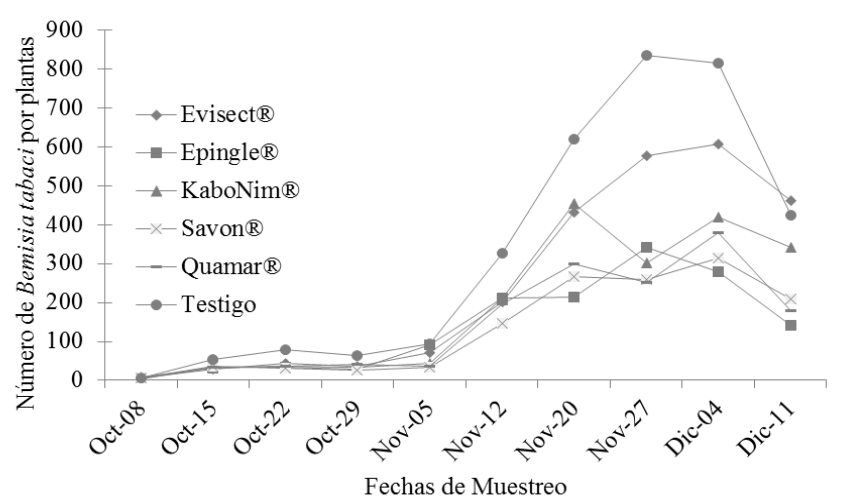

Figura 1: Fluctuación poblacional de Bemisia tabaci en el cultivo de tomate con los tratamientos evaluados de octubre a diciembre 2018, Estelí. Fuente: elaboración propia. 
Al realizar el análisis de varianza según Tukey, demostró que existen diferencias significativas ( $p \leq 0.0001$ ) entre los tratamientos evaluados, obteniendo que el menor número de Bemisia tabaci lo presentó el tratamiento Savon ${ }^{\circledR}$, con 132.65 Bemisia tabaci por planta, seguido del tratamiento Epingle ${ }^{\circledR}$, con 133.98 Bemisia tabaci y el tratamiento Quamar $^{\circledast}$ con 145.70 Bemisia tabaci por planta no encontrando diferencias significativas entre ambos. La mayor presencia de Bemisia tabaci por planta se refleja en el testigo (agua) con 331.33 Bemisia tabaci por planta (Cuadro 2).

Cuadro 2: Análisis de varianza de la fluctuación poblacional de Bemisia tabaci en el cultivo de tomate, con tratamientos evaluados de octubre a diciembre 2018 Estelí

\begin{tabular}{|c|c|c|c|}
\hline Tratamiento & Bemisia tabaci/planta Media & & Error estándar \\
\hline Savon $^{\circledR}$ & 132.65 & \pm & $21.23 a$ \\
\hline Epingle $^{\circledast}$ & 133.98 & \pm & $19.52 \mathrm{a}$ \\
\hline Quamar ${ }^{\circledast}$ & 145.70 & \pm & $21.38 \mathrm{a}$ \\
\hline Kabonim $^{\circledR}$ & 188.65 & \pm & $30.03 \mathrm{ab}$ \\
\hline Evisect $^{\oplus}$ & 246.63 & \pm & $40.06 \mathrm{~b}$ \\
\hline Testigo & 331.33 & \pm & $54.28 \mathrm{C}$ \\
\hline $\mathrm{N}$ & 240 & & \\
\hline CV & 31.58 & & \\
\hline$(F ; d f ; P)$ & $18.64,222,0.0001$ & & \\
\hline
\end{tabular}

ES: Error estándar, CV: Coeficiente de variación, N: Número de datos utilizados en el análisis,

F: Fischer calculado, df: Grado de libertad del error, P: Probabilidad según Tukey. Fuente: elaboración propia.

\section{Incidencia del daño de virosis transmitida por Bemisia tabaci, en el cultivo de tomate en tratamientos evaluados de octubre a diciembre del 2018, Estelí}

Se calculó el porcentaje de incidencia de daño de virosis transmitida por Bemisia tabaci en tratamientos Evisect ${ }^{\circledR}$, Epingle ${ }^{\circledR}$, Kabonim $^{\circledR}$, Savon ${ }^{\circledR}$, Quamar $^{\circledR}$ y Testigo, en el periodo comprendido entre octubre a diciembre de 2018, Estelí, desde los $05 \mathrm{ddt}$ hasta los $65 \mathrm{ddt}$. La incidencia de virosis se empezó a presentar en todos tratamientos a los $34 \mathrm{ddt}$ (noviembre 5), presentando mayor incidencia los tratamientos Testigo, Epingle ${ }^{\circledR}$ y Evisect ${ }^{\circledR}$, con un porcentaje de 15, 12.5 y 5. A los $65 \mathrm{ddt}$ los tratamientos que presentaron mayor incidencia de virosis fueron Testigo, Quamar ${ }^{\circledR}$ y Evisect ${ }^{\circledR}$, con un porcentaje del 65, 30 y 25. Esto se debe a que el testigo era absoluto, lo que permitió que B. tabaci trasmitiera el virus con facilidad, ya que en la planta no había ningún tipo de protección para insectos (Figura 2). 


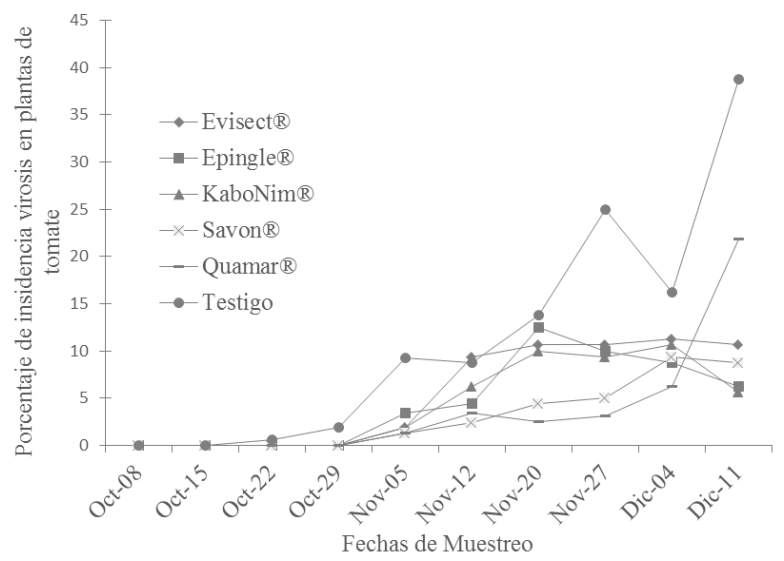

Figura 2: Incidencia del daño de virosis transmitida por mosca blanca Bemisia tabaci, en el cultivo de tomate, en tratamientos evaluados de octubre a diciembre del 2018, Estelí. Fuente: elaboración propia.

\section{Severidad del daño de virosis transmitida por Bemisia tabaci, en el cultivo de tomate en tratamientos evaluados de octubre a diciembre del 2018, Estelí}

Se comparó el porcentaje de severidad de daño de virosis transmitida por mosca blanca en los tratamientos Evisect ${ }^{\oplus}$, Epingle $^{\circledast}$, Kabonim $^{\circledR}$, Savon ${ }^{\circledast}$, Quamar ${ }^{\circledast}$ y Testigo en el periodo comprendido entre octubre a diciembre de 2018, Estelí, desde los 5 ddt hasta los $65 \mathrm{ddt}$. La severidad de virosis se presenta en todos los tratamientos a los 34 ddt (noviembre 5), con mayor severidad los tratamientos Testigo, Epingle ${ }^{\circledR}$, Evisect ${ }^{\circledR}$ y Kabonim ${ }^{\circledR}$ con un porcentaje de severidad 9.2, 3.3 y 1.8, respectivamente. A los $65 \mathrm{ddt}$ los tratamientos que presentaron mayor severidad de virosis fueron Testigo, Quamar ${ }^{\circledR}$ y Evisect ${ }^{\circledR}$, con un porcentaje del 38.7, 21.8 y 10.6, respectivamente (Figura 3).

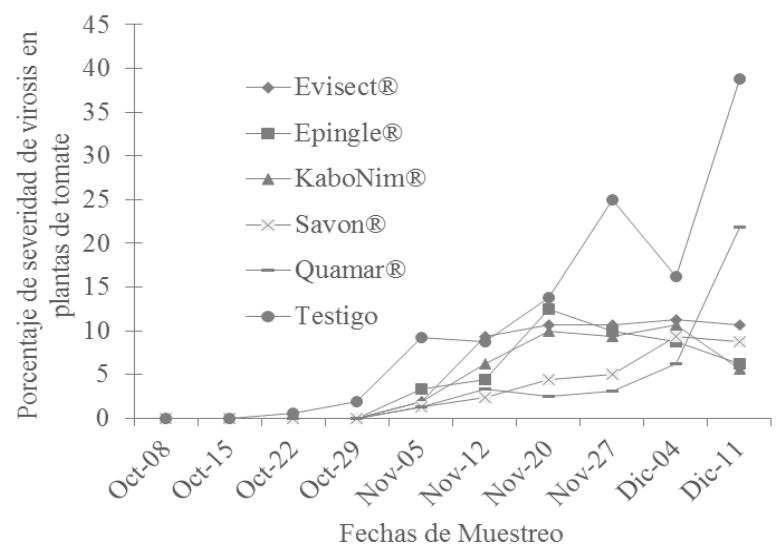

Figura 3: Severidad del daño de virosis transmitida por mosca blanca Bemisia tabaci, en el cultivo de tomate, en tratamientos evaluados de octubre a diciembre del 2018, Estelí. 
Jiménez Martínez (2012), asegura que Bemicia tabaci causa daños directamente al cultivo de tomate por ser trasmisora de Geminivirus, capaz de hacer distorsión en una plantación.

\section{Rendimiento total ( $\mathrm{kg} / \mathrm{ha}^{\mathrm{T}}$ ) de las parcelas de tomate en los tratamientos evaluados de octubre a diciembre de 2018, Estelí}

Se comparó el rendimiento total en $\mathrm{kg} / \mathrm{ha}^{1}$ de las parcelas de tomate en los tratamientos evaluados en el período de octubre a diciembre de 2018. Los rendimientos totales obtenidos muestran que el tratamiento que obtuvo el mayor rendimiento fue la parcela tratada con Epingle ${ }^{\circledR}$ con $44,057.77 \mathrm{~kg} / \mathrm{ha}^{1}$. El tratamiento Kabonim ${ }^{\circledR}$ con $34,469.70 \mathrm{~kg} / \mathrm{ha}$. Los tratamientos tratados con Quamar ${ }^{\circledR}$, Evisect ${ }^{\circledR}$ y Savon ${ }^{\circledR}$ obtuvieron rendimientos de 29,784.75, 29,403.41 y $28,527.46 \mathrm{~kg} / \mathrm{ha}^{1}$, respectivamente; en cambio el tratamiento Testigo obtuvo $25,047 \cdot 35 \mathrm{~kg} / \mathrm{ha}^{1}$ (Figura 4 ).

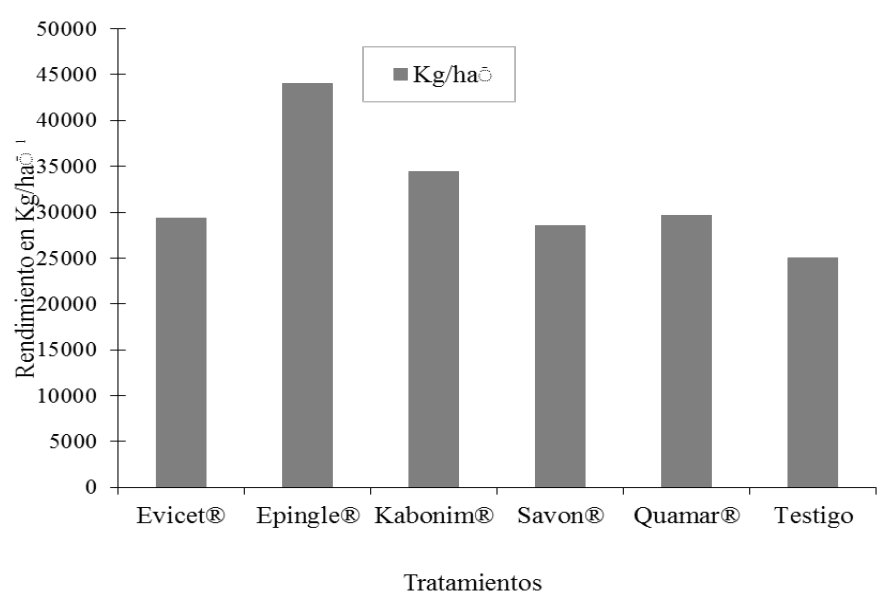

Figura 4: Rendimiento $\mathrm{kg} / \mathrm{ha}^{-1}$ de tomate. Fuente: elaboración propia.

Según Rodríguez y Morales (2007), obtuvieron los mejores rendimientos por hectárea al usar insecticidas químicos Gaucho ${ }^{\circledR}$ - Confidor ${ }^{\circledR}$ con $16,154.16 \mathrm{~kg} / \mathrm{ha}^{1}$, y el rendimiento más bajo lo obtuvieron al usar neem, con $9,783.03 \mathrm{~kg} / \mathrm{ha}^{1}$. Lo que difiere con este estudio, pues los mejores rendimientos se obtuvieron al usar Epingle ${ }^{\circledR} 44$ $057.77 \mathrm{~kg} / \mathrm{ha}^{1}$, seguido de Kabonim ${ }^{\circledR}$ con $34469.70 \mathrm{~kg} / \mathrm{ha}{ }^{1}$ y el rendimiento más bajo lo presentó el Testigo, con $25047.35 \mathrm{~kg} / \mathrm{h}^{1}$.

\section{Análisis económico de los tratamientos evaluados}

\section{Presupuesto parcial}

El análisis del presupuesto parcial, realizado según la metodología del CIMMYT 1998, determinó que los mayores costos variables los obtuvieron los tratamientos 
Epingle ${ }^{\circledR}$, Savon ${ }^{\circledR}$ y Evisect ${ }^{\circledR}$ con 73,68 y 67 US\$ $\$$ ha $^{1}$; los de menor costos variables fueron los tratamientos Testigo y Quamar ${ }^{\circledR}$ y Kabonim ${ }^{\circledR}$ con 27,50 y 52 US $\$ / h^{1}{ }^{1}$. El tratamiento que obtuvo el mayor beneficio neto fue Epingle ${ }^{\circledR}$ con 11677.53 US $\$ / \mathrm{ha}^{1}$, en cambio el tratamiento que presentó los menores beneficios netos fue Testigo con $5564.81 \mathrm{US} \$ / \mathrm{ha}^{1}$ (Cuadro 3).

Cuadro 3: Presupuesto parcial

\begin{tabular}{|c|c|c|c|c|c|c|}
\hline Concepto & Evisect $^{\oplus}$ & Epingle $^{\oplus}$ & Kabonim $^{\oplus}$ & Savon $^{\oplus}$ & Quamar $^{\oplus}$ & Testigo \\
\hline Total de CF US\$/ ha' & 2523.87 & 2523.87 & 2523.87 & 2523.87 & 2523.87 & 2523.87 \\
\hline Total de CV US\$/ ha' & 67 & 73 & 52 & 68 & 50 & 27 \\
\hline Beneficio neto US\$/ ha' & $6,936.15$ & $11,677.53$ & $8,592.32$ & $6,650.71$ & $7,060.56$ & $5,564.81$ \\
\hline
\end{tabular}

Fuente: elaboración propia.

Precio del producto al momento de la cosecha

Precio oficial del dólar U\$: 29.50

Precio al momento de la cosecha (o.44 US\$/kg)

\section{Conclusiones}

Los tratamientos Savon ${ }^{\circledR}$, Epingle ${ }^{\circledR}$ y Quamar ${ }^{\circledR}$ presentaron poblaciones bajas de Bemisia tabaci por planta.

La incidencia de virosis se presentó en todos los tratamientos a los 34 ddt, presentando menor incidencia los tratamientos, Kabonim ${ }^{\circledR}$, Savon ${ }^{\circledR}$, con 9.5\% y $10.5 \%$ por planta.

La severidad de virosis se presentó en todos los tratamientos a los $34 \mathrm{ddt}$, con menor severidad los tratamientos, Savon ${ }^{\circledR}$ y Quamar $^{\circledR}$, con el $3.11 \%$ y $3.84 \%$, respectivamente.

Los mayores rendimientos los alcanzaron los tratamientos Epingle ${ }^{\circledR}$ y Kabonim ${ }^{\circledR}$.

El análisis de presupuesto parcial nos indica que el Testigo fue el que presentó los menores costos variables y el tratamiento Epingle ${ }^{\circledR}$ fue el que presentó mayores costos variables. Los tratamientos Epingle ${ }^{\circledR}$ y Kabonim ${ }^{\circledR}$ fueron los que obtuvieron los beneficios netos mayores, por el contrario, el tratamiento que obtuvo el menor beneficio neto fue Testigo. 


\section{Lista de referencias}

Arysta, L. (2014). Ficha Técnica Evicet ${ }^{\circledR}$ s. Registro de venta ICA n² 2589. Bogota: ICA.

Atlántica. (2010a). Ficha Técnica de Kabonim. Managua: CISA.

Atlántica. (2010b). Ficha Técnica de Savon. Managua: CISA.

Atlántica. (2010c). Ficha técnica de Quamar. Managua: CISA.

Chavarría, S. (2004). Evaluación de cinco variedades de tomate (Lycopesicum esculentun Mill.) en relación al complejo de mosca blanca-geminivirus bajo infección natural en la zona del pacifico de Nicaragus. La Calera.

CIMMYT. (1988). La formulación de recomendaciones a partir de datos agronómicos. En Un manual metodológico de evaluación económica. DF México. https://repository. cimmyt.org/xmlui/bitstream/handle/10883/1063/9031.pdf

Di Rienzo, J., Balzarini, M., González, L., Casanoves., y Tablada, M. (2017). InfoStat versión Libre. Córdobas: UNC.

El Nuevo Diario. (21 de Diciembre de 2007). Comvocatoria. La Gaceta, págs. 246-247.

Escalano, E. A. (2009). Manual de cultivo de tomate. Chile: InnovaChile Corfo.

Foolad, M. R. (2012). Marker-Assisted Selection in Tomato Breeding. Critical Reviews in Plant Sciences, 31, 93-123. https://www.tandfonline.com/doi/abs/10.1080/ 07352689.2011 .616057

Hazera Genetics.(2015a). Ficha Técnica de Tomate. Managua: Agritrade.

Hazera Genetics. (2015b). Ficha Técnica de Tomate Orna. Managua: Agritrey.

Instituto Interamericano de Cooperación para la Agricultura. (2004). La contribución del IICA al desarrollo de la Agricultura y las comunidades Rurales de Nicaragua. Managua: IICA.

Instituto Nicaragüense de Fomento Municipal. (2000). Ficha del municipio de Estelí. Estelí: Alcaldia. 
Jiménez-Martínez, C. A. (2012). Manejo de mosca blanca (Bemisia tabaci Gennadius.) y geminivirus en semilleros de tomate (lycopersicum esculentum mill.) bajo protección física y química y su efecto en la producción. La Calera, 11(17), 05-13. https://repositorio.una.edu.ni/2361/1/pph10j61m.pdf

Programa de Manejo Integrado de Plagas para America Central. (2006). Nivel y Umbral de daño Económico de Plagas. Manual para el estudiante, 37-38.

Rodríguez, V., \& Morales, J. (2007). Evaluación de alternativas de protección físicas y químicas de semilleros de tomate ((Lycopersicum esculentum) contra el ataque del complejo de mosca blanca (Bemisia tabaci, Gennadius)-Geminivirus y su efecto en el rendimiento en el municipio de Tisma, Managua: CENIDA.

Sumitomo, C. (2008). Ficha Técnica de Epingle 10 EC (Pyriproxifen). Managua: Formunica. 